\title{
人工智能技术在电气自动化控制中的应用分析
}

\author{
郭俊材 赵伟刚 王昕悦 张田歌 \\ 洛阳矿山机械工程设计研究院有限责任公司，河南洛阳 471039
}

[摘要] 经济的进步, 催化了科技水平的提升, 在这样的背景下, 人工智能得到了较好的发展平台。无论是人工智能技术还是 电气自动化都属于较为先进的两种技术, 将两种技术进行融合, 可以实现行业的大跨度发展。在实际应用中, 在电气自动化 中融入人工智能的相关知识, 可以对成本进行有效、合理控制, 作用十分显著。基于此, 文章将重点研究人工智能的具体应 用, 以便进一步提高电气自动化水平, 为今后工作提供参考。

[关键词]自动化控制; 人工智能技术; 应用分析

DOI: $10.33142 /$ ec.v3i10.2704 中图分类号：TP3TN7 文献标识码：A

\section{Application Analysis of Artificial Intelligence Technology in Electric Automation Control}

GUO Juncai, ZHAO Weigang, WANG Xinyue, ZHANG Tiange

Luoyang Mining Machinery Engineering Design Institute Co., Ltd., Luoyang, Henan, 471039, China

\begin{abstract}
The progress of economy catalyzes the improvement of science and technology. In this context, artificial intelligence has got a better development platform. Both artificial intelligence technology and electrical automation belong to two advanced technologies. The integration of the two technologies can realize the long-span development of the industry. In practical application, the integration of artificial intelligence knowledge into electrical automation can effectively and reasonably control the cost. Based on this, this paper will focus on the specific application of artificial intelligence, in order to further improve the level of electrical automation and provide reference for future work.
\end{abstract}

Keywords: automatic control; artificial intelligence technology; application analysis

\section{引言}

在科学技术的推动下，人工智能发展势头强劲，发展空间巨大，为人工智能和自动化控制的融合奠定了基础，在 如今的自动化领域, 人工智能技术的应用频次越来越多, 将电气自动化水平推向了全新的高度。人工智能技术是在最 近几年兴起的一种新型技术，依靠思维模拟，创造了许多使用价值。在当今社会，信息科技的发展都少不了人工智能 的贡献, 可以对控制系统发展进行催化。

\section{1 应用优势}

\section{1 自动化操作的精度可以得到提升}

在科技和经济的推动下，人工智能得到了普及和飞速发展，将其应用到电气自动化领域具有一定的时代特征，优 势较为突出, 可以弥补电气自动化的不足, 促使电气自动化得以较好发展。电气自动化管理时, 有一些人为失误是经 常发生, 并且难以避免的, 这也是电气自动化发展的最大阻碍。为了将此类问题规避, 将人工智能引用进来, 可以实 现电气自动化的大跨度发展, 将自动化控制的能力全面提升, 确保理想的工作效率。在人工智能技术的影响下, 为电 气自动化的优化指明了方向, 就目前的情况看, 将人工智能应用到自动化控制中, 可以实现管理的升级换代, 改变传 统人工操作模式, 将人为失误的可能性降低, 将自动化控制精度大跨度提升, 可以从源头避免生产资源的浪费, 将成 本合理控制。在人工智能的应用阶段, 人工管理程度会大幅度降低, 许多工作都完成了人工和计算机的过渡, 计算机 被大量使用, 通过计算机可以对电气运转参数完成实时有效监测, 提升自动化控制水平, 提升自动化的精确度, 将错 误率控制在有效范围内, 降低错误发生的概率。除此之外, 在软硬件设施平稳运行的基础上, 电气自动化可以根据计 算机指令完成操作, 执行任务, 自动化操控更加合理有效, 精度更高, 可以全面提升电气自动化控制能力, 为行业发 展奠定基础。

\section{2 自动化操作方法变得更加简便}

除了可以提高自动化控制的可靠性与精确度之外, 人工智能的应用, 还可以使自动化操作方法变得更加简便。结 合现有的经验可以表明, 在自动化领域中尝试应用人工智能可以将生产效率大幅度提升, 想要实现这一目标, 需要依 靠人工智能的专家系统来实现，除此之外，还要配合模糊控制等，才能将自动化控制推向全新高度。为了在实际工作 
中可以将电气自动化的作用体现出来, 实现自动化和人工智能的高度融合, 专业系统发挥着关键作用。专业系统的功 能性是非常强大的, 在实际应用阶段, 通过正确指令输入, 专业系统就可以在较短时间内给出意见, 作用非常重大 ${ }^{[1]}$ 。 并且在整个过程中, 控制程序可以得到最大限度的优化, 同时自动化控制的手段也会比较简单, 控制的效率更高, 其 中模糊控制属于应用较多的控制方式, 将模糊控制理论应用到设备中, 就可以达到智能化控制目标, 将自动化控制水 平全面提升，管理更加精准和高效。

\section{2 具体应用}

\section{1 实现设备的精细化管理}

在经济发展的推动下, 市场竞争的激烈程度只增不减, 无论哪一类型的企业, 想要取得长足的进步与平稳发展, 就要想方设法找准自身优势, 将竞争力提升。通过精细化管理可以实现这一理想目标, 精细化管理可以帮助企业找到 自身发展中的不足, 实现企业运营成本的有效合理控制, 将企业运行质量全面提升, 这也是企业获取凝聚力和竞争力 的关键和重要保障。基于此, 在自动化控制中, 需要融入先进技术, 将智能化水平大幅度提升, 在生产阶段实现可靠 性运维。为了达到理想目标, 实现配电智能化, 将管理水平提升, 需要采用电能测量模块, 并配合使用多种智能元件, 提高控制水平, 完成实时能耗监测, 避免资源耗损严重, 可以通过远程运维的手段, 提高控制的智能化程度, 达到精 细化管理的理想目标，打造便捷运维体验。

\section{2 电气控制中的应用}

结合生产经验可以知道, 电气控制过程承担着重要职责, 属于核心环节, 其控制质量尤为重要, 是产品品质保证 的最主要因素。在实际应用中, 人工智能技术与电气控制的结合, 可以保障电气控制过程的实际效率。主要是因为人 工智能本身的技术性以及智能性, 采用该技术能够高效完成数据采集以及数据归类等工作。这种强大的数据处理能力 和人脑相比, 局限性较小, 应用的价值更高, 除此之外, 再搭配使用云计算功能, 在云计算的帮助下, 数据采集更加 可靠和高效, 可以短时间传递给数据终端, 为处理数据和运用数据提供了保障。数据达到计算机终端后, 可以自动完 成接下来的分类操作, 并在此基础上对数据价值进行分析, 将有效数据转化, 这些数据信息将会成为生产的必备资料, 确保数据供给的安全与可靠 ${ }^{[2]}$ 。另外, 人工智能在电气系统监管方面发挥着重要作用, 可以对电气仪表数值进行检测, 一旦发现异常, 便可以积极应对, 迅速反应, 并进行预警。倘若存在不正当操作行为, 系统或者是正在遭受着攻击, 人工智能可以起到很好的防护效果, 通过强行关闭电源等操作, 将事故发生的风险概率降低, 避免事故发生。基于人 工智能技术的强大功能以及其在自动化控制中的表现, 目前许多学者正在加大相关的研究力度, 主要是以模糊控制为 主, 对产业结构进行优化, 实现人工智能和产业发展的高度融合。

\section{3 解决相关故障问题}

除了在上述方面, 人工智能的应用较为广泛之外, 在解决相关故障问题方面, 人工智能也发挥出了巨大优势。结 合以往经验可以得出, 人工智能在自动化控制领域中的推广, 不仅可以全面优化工作质量和提升效率, 还可以实现故 障的快速查找, 提高故障查找效率, 促使系统可以长期处于高效、平稳运行的状态。在传统意义上的电气工程中, 如 果遇到运作问题, 相关工作人员则需要通过现场状况和自身经验对故障进行查找, 当然还要具备一定的故障检修技术, 才可以完成此项工作。但是采用这样的方法, 故障的排查效率是很难保障的, 为了进一步提升检修效率, 需要通过增 加人员的手段来解决。基于这样的现状, 人工智能的出现以及在自动化领域中的应用, 可以有效完成故障搜索工作, 为故障检修节省了大量时间, 为故障解决预留了充足时间, 通过人工智能技术, 电气工程的效率可以得到有效保障。 具体来说, 需要相关技术人员编写各个方面的参数, 这是工作的前提, 当出现故障时, 系统程序会自动检测, 根据故 障发生环节完成相关数据的报错。采用这种先进的报错手段, 可以明确故障发生的具体环节, 锁定故障位置, 帮助检 修人员快速解决故障，确保电气工程迅速恢复，将生产效率全面提升。

\section{3 结语}

综上可知, 由目前的情况看, 人工智能已经进入了新的发展阶段, 人工智能的应用, 可以达到产业效益提升的目 标。尤其是在电气自动化中的应用, 无论是精细化管理还是故障检测都发挥着关键作用, 地位逐渐突显, 随着人工智 能应用的深入, 将电气自动化推向了全新高度。发展与推广人工智能, 可以实现自动化产业的飞跃。

\section{[参考文献]}

[1]彭龙生.人工智能技术在电气自动化控制中的应用研究 [J].科技经济导刊, 2020 (25): 32-87.

[2]张坤平. 试论人工智能技术在电气自动化控制中的应用 [J]. 信息系统工程, 2020 (08): 84-85.

作者简介: 郭俊材 (1982.10-), 男, 毕业院校: 华中科技大学, 现就职单位: 洛阳矿山机械工程设计研究院有限责任 公司。 